\title{
IMPROVEMENT OF METHANE STORAGE CAPACITY IN ACTIVATED CARBON BED WITH BIDISPERSE PACKING
}

\author{
Jailson C. Santos ${ }^{1}$, João A. Lima², José M. Gurgel ${ }^{2}$ and Francisco Marcondes ${ }^{3 *}$ \\ ${ }^{1}$ Universidade Regional do Cariri, Departamento de Engenharia de Produção Mecânica, Juazeiro do Norte, CE, Brasil. ORCID: 0000-0001-7547-3097 \\ ${ }^{2}$ Universidade Federal da Paraíba, Centro de Energias Alternativas e Renováveis, Laboratório de Energia Solar, \\ João Pessoa, PB, Brasil. ORCID: 0000-0002-0698-9994; ORCID: 0000-0002-1801-2304 \\ ${ }^{3}$ Universidade Federal do Ceará, Departamento de Engenharia Metalúrgica e de Materiais, Fortaleza, CE, Brasil. \\ E-mail: marcondes@ufc.br-ORCID: 0000-0002-3010-5343
}

(Submitted: February 22, 2018 ; Revised: September 24, 2018 ; Accepted: October 27, 2018)

\begin{abstract}
This study presents a numerical investigation of the dynamics of methane adsorption in granular activated carbon beds with bidisperse packing in order to reduce the void spaces between the adsorbent particles and to improve the storage capacity in adsorbed natural gas vessels. Two distinct particle sizes (d and D) were used for the packing of the bidisperse bed, and the following particle size ratios were investigated: $\delta=d / D=$ 1 (monodisperse bed), 1/5,1/10,1/15, and 1/20. Compared with the monodisperse bed condition, the results obtained showed that bidisperse packing increases bed density by about $30 \%$ and methane storage capacity by up to $20 \%$. The results also showed that the charging time could be optimized by increasing the pressure drop applied to the bed and that the storage capacity can be increased by cooling the admitted gas in the vessel. Keywords: Adsorbed natural gas; Bidisperse bed; Storage capacity; Packing density; Numerical model; Fixed bed.
\end{abstract}

\section{INTRODUCTION}

The volatility of the oil market in conjunction with the environmental issues inherent in the exploitation of fossil fuels has led to the search for alternative fuels around the world. In this context, natural gas, an abundant fuel that is still little explored, appears as a promising source of energy for applications in automotive vehicles. Natural gas is cheaper than gasoline and diesel. It burns cleaner and therefore represents an interesting alternative for applications in large urban centers where air pollution is a serious public health problem. However, for natural gas to be used on a large scale, a safe and economical storage technology needs to be developed. The major drawback of the dissemination of natural gas in automotive vehicles is the fact that its storage and application under normal conditions of temperature and pressure is not feasible. This is because of its low volumetric energy density (defined as the heat of combustion per unit volume) of approximately 0.038 $\mathrm{MJ} / \mathrm{L}$, representing only $0.11 \%$ of the value obtained for gasoline (34.8 MJ/L) (Lozano-Castelló et al., 2002). This fact naturally results in low mileage per volume of tank and, consequently, low autonomy of natural gas compared to gasoline. Owing to their high volumetric energy density, liquefied natural gas and compressed natural gas technologies have traditionally been used for storing natural gas; however, these technologies are expensive and difficult to operate. In liquefied natural gas technology, the gas is stored at cryogenic temperatures that raises the operational cost and requires trained personnel to handle the cryogenic liquids. However, in compressed natural gas technology, the gas is stored at a high pressure (approximately $20 \mathrm{MPa}$ ) that requires multiple stages

\footnotetext{
* Corresponding author: Francisco Marcondes - E-mail: marcondes@ufc.br
} 
of compression and hence increases the costs and presents safety restrictions. An attractive technology that uses a single compression stage is adsorbed natural gas (ANG) technology, which is based on the use of an adsorbent with a high number of micropores to fix and accommodate the gas molecules. Given the high volume of micropores, many molecules are attracted to the surface of the adsorbent, resulting in a much higher density than that of the gas phase in equilibrium. In fact, ANG technology makes it possible to obtain high storage capacities in the order of $200 \mathrm{~V} / \mathrm{V}$ with pressures on the order of $3.5 \mathrm{MPa}$, which can be easily obtained with a single compression stage (Mota et al., 1997; Menon and Komarneni, 1998; LozanoCastelló et al., 2002; Rahman et al., 2011; Roszak and Chorowski, 2013; Kayal et al., 2015). Reducing the storage pressure reduces costs and facilitates the development of safer and lighter vessels. Activated carbon has been the most suitable adsorbent for ANG-vessel applications because of its high surface area and micropore volume; however, despite its advantages, ANG technology has some limitations. Adsorption is an exothermic phenomenon and, during rapid loading of natural gas under conditions in which adsorption heat is not dissipated into the environment, less methane is stored in the vessel. However, during the discharge process, the porous bed cools and an additional amount of natural gas remains in the vessel at exhaustion pressure. Several researchers have pointed out different solutions to limit temperature fluctuations in ANG vessels during charge and discharge steps (Chang and Talu, 1996; Vasiliev et al., 2000; Mota et al., 2004; Yang et al., 2005; Santos et al., 2009; Santos et al., 2014; Santos et al., 2015) .

The performance of an ANG system is often evaluated through a parameter known as delivery. Delivery is defined as the volume of gas released per unit volume of a vessel and is designated by $\mathrm{V} / \mathrm{V}$. In applications where the volume available for accommodation of the ANG vessel represents a limitation, as in the case of automotive vehicles, it is crucial that the amount of gas released per unit volume of a vessel is as large as possible. The United States Department of Energy (USDOE) recently established a minimum delivery of $263 \mathrm{~V} / \mathrm{V}$ for an ANG system operating at a pressure of $3.5 \mathrm{MPa}$ and temperature of $25^{\circ} \mathrm{C}$ to be considered economically viable (Prajwal and Ayappa, 2014). The minimum delivery represents an important challenge in the search for a feasible ANG technology. Although activated carbon presents a high capacity for adsorption on a mass basis, it does not do so on a volumetric base because of its low packing density. This fact naturally results in low volumetric storage capacity and delivery (V/V).

According to Mota and Lyubchik (2008), there are four distinct zones in an ANG vessel where the gas density is substantially different: 1) the volume of voids between the particles where the gas is compressed at the reservoir pressure; 2) the macroporous and mesoporous volumes of the adsorbent, where the density of the gas is not significantly higher than that of the compressed gas in the voids between the particles; 3) the volume occupied by the solid matrix that does not contribute to the gas storage; and 4) the microporous volume of the adsorbent where the largest amount of gas is located. The higher density of the adsorbed gas in the micropores should compensate for the volume taken up by the lower density of the gas in the voids between the particles and in the macro and mesopores of the adsorbent. It is observed, therefore, that the maximization of the storage capacity depends on the maximization of the volume of micropores and the reduction of voids between the particles and in the macro and mesopores of the adsorbent. The maximization of micropores and reduction of macropores/mesopores depends on the adsorbent manufacturing process, while the reduction of voids between the particles can be achieved with efficient packing methods.

The efficient packing of the porous bed offers the added benefit of an increase in the effective thermal conductivity of the bed, thereby enhancing the process of heat transfer in an ANG vessel. An effective way of reducing voids in an activated carbon bed and increasing the packing density is to promote packing with particles of multiple sizes so that the small particles occupy the voids between the large particles. According to Talu (1992), packing with particles of two different sizes (bidisperse packing) can raise the packing density by up to $30 \%$ and increase the volumetric storage capacity of an ANG vessel. Few studies have been published to investigate efficient forms of packing in ANG vessels; a few available studies were published in the patent form (Greenbank, 1990; Greenbank, 1992; Chang, 1994). Although raising the packing density of ANG vessels is desirable, reducing the porosity of the bed can greatly reduce the permeability of a vessel and lead to very long charge and discharge times. In this sense, there is a clear need to optimize the particle size ratio to maximize volumetric storage capacity with acceptable loading and unloading times. The optimization of the particle size ratio used in an ANG vessel can be done by developing a model for the heat and mass transfer processes in the bidisperse bed. To the best of our knowledge all works that deal with the modeling of the loading process in ANG vessels consider only monodisperse packing. Therefore, the contribution of the present study is the development of a numerical model to optimize the loading process of ANG vessels with bidisperse packing. It is intended to investigate the optimum ratio between adsorbent particle sizes to achieve maximum volumetric storage 
capacity in $\mathrm{V} / \mathrm{V}$ with the achievement of filling times that are acceptable for fast-loading applications.

\section{POROSITY AND PACKING DENSITY IN BIDISPERSE BEDS}

Figure 1 shows the ANG vessel constituted of several columns of granular activated carbon with bidisperse packing used in the study. It is assumed that the flow in each column has the same behavior. Therefore, we choose a single column (adsorbent bed) to study the adsorption process. The column wall is made of steel and therefore there is not mass transfer to the external air. The bed was compacted with spherical adsorbent particles of two different sizes. The reduction of interparticle porosity and the increase in packing density were obtained when the small particles were inserted into the voids between the large particles. Typically, the method of constructing a particle bed of a distinct size within a reservoir involves creating a mixture of components of chosen proportions outside the reservoir, which is then inserted into the reservoir. In the final step, the particles are compacted. Different packing methods are discussed in the literature (Dias et al., 2004), including rotation and vibration.

In Fig. 1, the total volume of the adsorbent column is given by the sum of the volume of the voids between the particles $\left(V_{V}\right)$, the volume occupied by the large particles $\left(V_{D}\right)$, and the volume occupied by the small particles $\left(V_{d}\right)$.

$\mathrm{V}_{\mathrm{T}}=\mathrm{V}_{\mathrm{V}}+\mathrm{V}_{\mathrm{D}}+\mathrm{V}_{\mathrm{d}}$

The interparticle porosity of the bed $(\varepsilon)$ is defined as the ratio of the volume of voids between the particles and the total volume (volume of interparticle voids plus the volume of small and large particles). The fractional porosity of the large particles $\left(\varepsilon_{D}\right)$ is defined as the fraction of voids of the large particles in the total volume of the mixture, while the fractional porosity $\left(\varepsilon_{d}\right)$ denotes the void fraction of the small particles in the remaining void volume of the mixture (Dias et al., 2004). Therefore, the interparticle porosity $(\varepsilon)$ and the fractional porosities $\left(\varepsilon_{D}\right)$ and $\left(\varepsilon_{d}\right)$ are respectively, defined as:

$$
\begin{aligned}
& \varepsilon=\frac{V_{V}}{V_{T}}=\frac{V_{T}-V_{D}-V_{d}}{V_{T}}=1-\frac{V_{D}+V_{d}}{V_{T}} \\
& \varepsilon_{D}=\frac{V_{V}+V_{d}}{V_{T}}=\frac{V_{T}-V_{D}}{V_{T}}=1-\frac{V_{D}}{V_{T}} \\
& \varepsilon_{d}=\frac{\left(V_{T}-V_{D}\right)-V_{d}}{V_{T}-V_{D}}=1-\frac{V_{d}}{V_{T}-V_{D}}=1-\frac{V_{d}}{\varepsilon_{D} V_{T}}
\end{aligned}
$$

After some algebraic manipulations of Eqs. (2), (3), and (4) we obtain the following relationship between the porosities of the bidisperse bed:

$\varepsilon_{\mathrm{d}}=\frac{\varepsilon}{\varepsilon_{\mathrm{D}}}$

As described by Dias et al. (2004), the volume fraction of large particles in the total volume of particles in the mixture $\left(x_{D}\right)$ is defined by

$$
\mathrm{x}_{\mathrm{D}}=\frac{\mathrm{V}_{\mathrm{D}}}{\mathrm{V}_{\mathrm{D}}+\mathrm{V}_{\mathrm{d}}}
$$

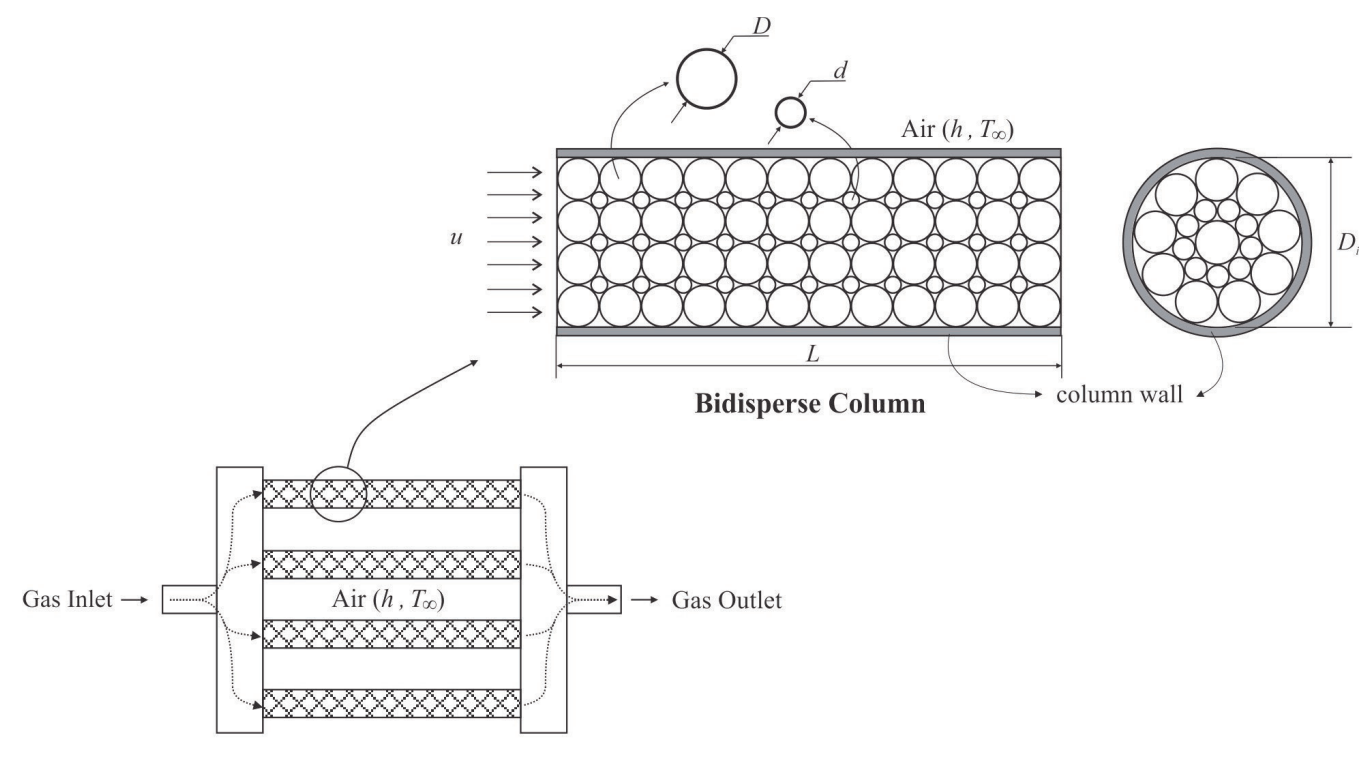

ANG Vessel

Figure 1. ANG vessel using columns of activated carbon with bidisperse packing. 
Manipulating Eqs. (2), (3), and (6) we have the following relationship between $\varepsilon_{\mathrm{D}}$ and $\mathrm{x}_{\mathrm{D}}$ :

$\varepsilon_{\mathrm{D}}=1-(1-\varepsilon) \mathrm{x}_{\mathrm{D}}$

Dias et al. (2008) developed a method to determine the interparticle porosity in polydisperse beds (several particle sizes). The interparticle porosity of binary mixtures with particles of two different sizes depends on the ratio of the diameter of the small particles to the large particles, $\delta=d / D$, and the large particle volumetric fraction $\left(x_{D}\right)$, which is defined as the ratio of the volume occupied by the large particles to the total volume of the solid in the reservoir. Dias et al. (2008) proposed the following equation to describe the interparticle porosity of bidisperse beds:

$\varepsilon=\frac{\varepsilon_{\mathrm{d}}^{0}\left(1-\mathrm{x}_{\mathrm{D}}\right) \exp \left(1.2264 \mathrm{x}_{\mathrm{D}}{ }^{1 / \sqrt{\delta}}\right)}{1-\varepsilon_{\mathrm{d}}^{0} \mathrm{x}_{\mathrm{D}}}$

where $\varepsilon_{d}^{0}$ denotes the porosity of the monodisperse bed obtained with the packing of the small-sized particles.

According to Dias et al. (2008), for the bidisperse beds, the value of the volumetric fraction of large particles, for which the porosity is minimal, $x_{D \min }$, is approximately 0.7 . As the aim of our study is to minimize the voids in the adsorbent bed to maximize the packing density, $x_{D}=0.7$ will be considered in Eq. (8), while the particle size ratio $\delta$ will be variable.

The packing density is important to evaluate the volumetric storage capacity and delivery of ANG vessels (Rios et al., 2011). We present now a procedure to evaluate this parameter. The packing density of the bed $\left(\rho_{b}\right)$ is defined by the ratio of the mass of solid packed in the reservoir $\left(\mathrm{m}_{\mathrm{S}}\right)$ to the total volume.

$\rho_{\mathrm{b}}=\frac{\mathrm{m}_{\mathrm{S}}}{\mathrm{V}_{\mathrm{T}}}=\frac{\rho_{\mathrm{S}} \mathrm{V}_{\mathrm{S}}}{\mathrm{V}_{\mathrm{T}}}=\frac{\rho_{\mathrm{S}}\left(\mathrm{V}_{\mathrm{SD}}+\mathrm{V}_{\mathrm{Sd}}\right)}{\mathrm{V}_{\mathrm{T}}}$

where $\rho_{S}$ and $V_{S}$ denote, respectively, the adsorbent density and total volume of solid in the bed. The total volume of solid in the bed, $V_{s}$, is obtained by summing the volume of the solid part within the large particles, $V_{S D}$, with the volume of the solid part in the small particles, $V_{S d}$.

Since the bed particles are porous, the volume of all particles can be obtained by summing the volume of the solid part within the particles with their void volume (intraparticle). Therefore, for the large particles, we have:

$\mathrm{V}_{\mathrm{D}}=\mathrm{V}_{\mathrm{VD}}+\mathrm{V}_{\mathrm{SD}}$ where $V_{V D}$ and $V_{S D}$ denote the void volume and the volume of the solid part of the large particles, respectively. Similarly, for the small particles, we have

$\mathrm{V}_{\mathrm{d}}=\mathrm{V}_{\mathrm{Vd}}+\mathrm{V}_{\mathrm{Sd}}$

Substituting Eqs. (10) and (11) into Eq. (1), we obtain:

$\mathrm{V}_{\mathrm{T}}=\mathrm{V}_{\mathrm{V}}+\mathrm{V}_{\mathrm{VD}}+\mathrm{V}_{\mathrm{SD}}+\mathrm{V}_{\mathrm{Vd}}+\mathrm{V}_{\mathrm{Sd}}$

Substituting Eq. (12) into Eq. (9), the following result is obtained:

$\rho_{\mathrm{b}}=\frac{\rho_{\mathrm{S}}\left(\mathrm{V}_{\mathrm{T}}-\mathrm{V}_{\mathrm{V}}-\mathrm{V}_{\mathrm{VD}}-\mathrm{V}_{\mathrm{Vd}}\right)}{\mathrm{V}_{\mathrm{T}}}=\rho_{\mathrm{S}}\left(1-\frac{\mathrm{V}_{\mathrm{V}}}{\mathrm{V}_{\mathrm{T}}}-\frac{\mathrm{V}_{\mathrm{VD}}}{\mathrm{V}_{\mathrm{T}}}-\frac{\mathrm{V}_{\mathrm{Vd}}}{\mathrm{V}_{\mathrm{T}}}\right)$

Substituting Eq. (3) into Eq. (2), we obtain:

$\frac{\mathrm{V}_{\mathrm{d}}}{\mathrm{V}_{\mathrm{T}}}=\varepsilon_{\mathrm{D}}-\varepsilon$

After some algebraic work, Eq. (3) can be written as:

$\frac{\mathrm{V}_{\mathrm{D}}}{\mathrm{V}_{\mathrm{T}}}=1-\varepsilon_{\mathrm{D}}$

The intraparticle porosity of the large particle $\left(\varepsilon_{p D}\right)$ and intraparticle porosity of the small particle $\left(\varepsilon_{p d}\right)$ are, respectively, given by:

$$
\begin{gathered}
\varepsilon_{\mathrm{pD}}=\frac{\mathrm{V}_{\mathrm{VD}}}{\mathrm{V}_{\mathrm{D}}} \\
\varepsilon_{\mathrm{pd}}=\frac{\mathrm{V}_{\mathrm{Vd}}}{\mathrm{V}_{\mathrm{d}}}
\end{gathered}
$$

Substituting Eqs. (2), (16), and (17) into Eq. (13), we obtain:

$\rho_{\mathrm{b}}=\rho_{\mathrm{S}}\left(-\varepsilon-\frac{\varepsilon_{\mathrm{pD}} \mathrm{V}_{\mathrm{D}}}{\mathrm{V}_{\mathrm{T}}}-\frac{\varepsilon_{\mathrm{pd}} \mathrm{V}_{\mathrm{d}}}{\mathrm{V}_{\mathrm{T}}}\right)$

Finally, replacing Eqs. (14) and (15) into Eq. (18), the packing density of the bidisperse bed shown in Fig. 1 is given by:

$\rho_{\mathrm{b}}=\rho_{\mathrm{S}}\left[(1-\varepsilon)-\varepsilon_{\mathrm{pD}}\left(1-\varepsilon_{\mathrm{D}}\right)-\varepsilon_{\mathrm{pd}}\left(\varepsilon_{\mathrm{D}}-\varepsilon\right)\right]$

\section{MATHEMATICAL MODEL}

Figure 1 shows the ANG vessel considered. The storage vessel is formed by several columns 
compacted with activated carbon. Assuming that each column has a similar performance, we investigated a single column filled with activated carbon open at both ends. For all results presented in this work, the internal diameter and length of each column are equal to $5 \mathrm{~cm}$ and $0.5 \mathrm{~m}$, respectively. Therefore, we neglected the thermal and mass gradients in the radial direction. The column was filled with adsorbent particles of two different sizes to obtain a bidisperse packing. The gas flows from one end of the column to the other. Next, we show the equations that describe the dynamics of methane adsorption in the bidisperse column along with the boundary and initial conditions.

\section{Column model}

For the column model, the following assumptions were made:

- $\quad$ radial effects are negligible;

- natural gas is constituted of pure methane; and

- $\quad$ sorbate behaves as an ideal gas.

The gas flow is described by the mass, momentum, and energy balances, and the ideal gas equation, respectively, as described below:

$$
\begin{aligned}
& \frac{\partial \rho_{\mathrm{f}}}{\partial \mathrm{t}}+\frac{\partial}{\partial \mathrm{x}}\left(\rho_{\mathrm{f}} \mathrm{u}\right)=-\frac{\left(1-\varepsilon_{\mathrm{d}}\right) \varepsilon_{\mathrm{D}}}{\varepsilon} \frac{\partial \overline{\mathrm{q}}_{\mathrm{d}}}{\partial \mathrm{t}}-\frac{\left(1-\varepsilon_{\mathrm{D}}\right)}{\varepsilon} \frac{\partial \overline{\mathrm{q}}_{\mathrm{D}}}{\partial \mathrm{t}} \\
& \frac{\partial}{\partial \mathrm{t}}\left(\rho_{\mathrm{f}} \mathrm{u}\right)+\frac{\partial}{\partial \mathrm{x}}\left(\rho_{\mathrm{f}} \mathrm{u} \mathrm{u}\right)=-\frac{\partial \mathrm{p}}{\partial \mathrm{x}}-\frac{\mu \varepsilon \mathrm{u}}{\mathrm{K}}-\frac{\mathrm{C}_{\mathrm{F}} \rho_{\mathrm{f}} \varepsilon^{2} \mathrm{u}^{2}}{\sqrt{\mathrm{K}}} \\
& \frac{\partial}{\partial \mathrm{t}}\left(\rho_{\mathrm{f}} \mathrm{T}_{\mathrm{f}}\right)+\frac{\partial}{\partial \mathrm{x}}\left(\rho_{\mathrm{f}} \mathrm{u} \mathrm{T}_{\mathrm{f}}\right)=\frac{\partial}{\partial \mathrm{x}}\left(\frac{\lambda_{\mathrm{f}}}{\mathrm{cp}_{\mathrm{f}}} \frac{\partial \mathrm{T}_{\mathrm{f}}}{\partial \mathrm{x}}\right)+ \\
&+\frac{6 \mathrm{~h}_{\mathrm{pd}}}{\mathrm{d}} \frac{\left(1-\varepsilon_{\mathrm{d}}\right) \varepsilon_{\mathrm{D}}}{\varepsilon \mathrm{cp}_{\mathrm{f}}}\left(\mathrm{T}_{\mathrm{sd}}-\mathrm{T}_{\mathrm{f}}\right)+ \\
&+\frac{6 \mathrm{~h}_{\mathrm{pD}}}{\mathrm{D}} \frac{\left(1-\varepsilon_{\mathrm{D}}\right)}{\varepsilon \mathrm{cp}_{\mathrm{f}}}\left(\mathrm{T}_{\mathrm{sD}}-\mathrm{T}_{\mathrm{f}}\right)+ \\
&+\frac{2 \mathrm{U}_{\mathrm{g}}\left(\mathrm{T}_{\infty}-\mathrm{T}_{\mathrm{f}}\right)}{\varepsilon \mathrm{R}_{\mathrm{i}} \mathrm{cp}_{\mathrm{f}}}
\end{aligned}
$$

$\rho_{\mathrm{f}}=\frac{p}{\mathrm{R}_{\mathrm{g}} \mathrm{T}_{\mathrm{f}}}$

In Eq. (20), the first term on the right-hand side represents the mass transfer of gas to the small adsorbent particles, while the second term represents the mass transfer to the large adsorbent particles. In Eq. (21), $K$ denotes the permeability of the porous media. Several studies addressing the permeability of a porous bed consisting of a mixture of particles with multiple sizes are available in the literature (Ben Aim et al., 1971; Standish and Mellor, 1980; Standish and Collins, 1983; Leitzelement et al., 1985; MacDonald et al., 1991; Mota et al., 2001; Dias et al., 2007; Dias et al., 2008). In this work, we use the model given by Dias et al. (2008) to evaluate the permeability of the bidisperse bed. The parameter $C_{F}$ was chosen in order for the momentum equation to reproduce Ergun's equation when the steady state is reached. These two parameters are given by:

$$
\begin{aligned}
& \mathrm{K}=\frac{\left(\mathrm{d}_{\mathrm{av}}\right)^{2} \varepsilon^{2}}{\left(36 \mathrm{~K}_{0} \tau^{2}(1-\varepsilon)^{2}\right)} \\
& \mathrm{C}_{\mathrm{F}}=\frac{1.75 \varepsilon^{-3 / 2}}{\sqrt{150}}
\end{aligned}
$$

In Eq. (24), $d$ represents the mean diameter of the particles used in the bidisperse packing (Dias et al., 2008), $K_{0}$ was assumed equal to $150 / 36$ to satisfy Ergun's equation, and $\tau$ denotes the tortuosity of the porous media. Finally, in Eq. (22), the last three terms on the right-hand side represent the heat transfer from the gas to the small particles, the heat transfer to the large particles, and the heat exchange between the column and environment, respectively.

To study the charge process in the bidisperse bed, it was assumed that gas with a prescribed pressure $\left(p_{\text {in }}\right)$ and temperature $\left(T_{i n}\right)$ is suddenly forced into the column, while the outlet pressure is kept constant at the initial pressure $\left(p_{0}\right)$. The column is initially saturated with gas at pressure $\mathrm{p}_{0}$ and temperature $T_{0}$. These values correspond to the last values obtained in the desorption process. The initial condition for the velocity field is $u_{0}=0$. Therefore, the initial and boundary conditions for the bed equations are given by:

$$
\begin{aligned}
& \mathrm{p}(\mathrm{x}, \mathrm{t}=0)=\mathrm{p}_{0} ; \mathrm{T}_{\mathrm{f}}(\mathrm{x}, \mathrm{t}=0)=\mathrm{T}_{0} ; \mathrm{u}(\mathrm{x}, \mathrm{t}=0)=\mathrm{u}_{0} \\
& \mathrm{p}(\mathrm{x}=0, \mathrm{t})=\mathrm{p}_{\text {in }} ; \mathrm{T}_{\mathrm{f}}(\mathrm{x}=0, \mathrm{t})=\mathrm{T}_{\text {in }} \\
& \mathrm{p}(\mathrm{x}=\mathrm{L}, \mathrm{t})=\mathrm{p}_{0} ; \frac{\partial \mathrm{T}_{\mathrm{f}}(\mathrm{x}=\mathrm{L}, \mathrm{t})}{\partial \mathrm{x}}=0
\end{aligned}
$$

\section{Pellet model}

The following assumptions were made regarding the adsorbent pellets:

- pellets are uniformly distributed spherical particles;

- the mass transfer in the adsorbent particles is described by the monodisperse model, while the effective mass diffusion coefficient is constant;

- temperature is uniform inside the adsorbent particles. Our investigation will be performed for five particle ratios $(d / D=1,1 / 5,1 / 15,1 / 20)$. We kept $d$ equal to $0.5 \mathrm{~mm}$ with $\mathrm{D}$ variable. The worst situation 
for the assumption of uniform temperature is for $\mathrm{d} / \mathrm{D}$ equal to $1 / 20$; the Biot number for this particle ratio is equal to 0.6 , which could result in some temperature variation inside the particle.

- adsorption equilibrium is attained in the external surface of the adsorbent particles.

The adsorbent bed is formed by the packing of large and small particles; therefore, the heat and mass transfer processes should be described separately for the two types of particles since the kinetics of sorption depend on the size of the pellets. For the large pellets, the mass and energy balances are given by:

$\frac{\partial \mathrm{q}_{\mathrm{D}}}{\partial \mathrm{t}}=\frac{1}{\mathrm{r}^{2}} \frac{\partial}{\partial \mathrm{r}}\left(\mathrm{r}^{2} \mathrm{D}_{\mathrm{ef}} \frac{\partial \mathrm{q}_{\mathrm{D}}}{\partial \mathrm{r}}\right)$

$\mathrm{C}_{\mathrm{S}} \frac{\partial \mathrm{T}_{\mathrm{SD}}}{\partial \mathrm{t}}=\frac{6 \mathrm{~h}_{\mathrm{pD}}}{\mathrm{D}}\left(\mathrm{T}_{\mathrm{f}}-\mathrm{T}_{\mathrm{SD}}\right)+(-\Delta \mathrm{H}) \frac{\partial \overline{\mathrm{q}}_{\mathrm{D}}}{\partial \mathrm{t}}$

The following are initial and boundary conditions for Eqs. (29) and (30):

$\mathrm{q}_{\mathrm{D}}(\mathrm{r}, \mathrm{t}=0)=\mathrm{q}^{*}\left(\mathrm{p}_{0}, \mathrm{~T}_{0}\right) ; \mathrm{T}_{\mathrm{SD}}(\mathrm{t}=0)=\mathrm{T}_{0}$

$\frac{\partial \mathrm{q}_{\mathrm{D}}(\mathrm{r}=0, \mathrm{t})}{\partial \mathrm{r}}=0$

$\mathrm{q}_{\mathrm{D}}(\mathrm{r}=\mathrm{D} / 2, \mathrm{t})=\mathrm{q}^{*}\left(\mathrm{p}, \mathrm{T}_{\mathrm{SD}}\right)$

where $\mathrm{q}^{*}$ is relative to the adsorption equilibrium. For the small pellets, the mass and energy balances are similar to those given for the large pellets.

$\frac{\partial \mathrm{q}_{\mathrm{d}}}{\partial \mathrm{t}}=\frac{1}{\mathrm{r}^{2}} \frac{\partial}{\partial \mathrm{r}}\left(\mathrm{r}^{2} \mathrm{D}_{\mathrm{ef}} \frac{\partial \mathrm{q}_{\mathrm{d}}}{\partial \mathrm{r}}\right)$

$\mathrm{C}_{\mathrm{S}} \frac{\partial \mathrm{T}_{\mathrm{Sd}}}{\partial \mathrm{t}}=\frac{6 \mathrm{~h}_{\mathrm{pd}}}{\mathrm{d}}\left(\mathrm{T}_{\mathrm{f}}-\mathrm{T}_{\mathrm{Sd}}\right)+(-\Delta \mathrm{H}) \frac{\partial \overline{\mathrm{q}}_{\mathrm{d}}}{\partial \mathrm{t}}$

The initial and boundary conditions for Eqs. (34) and (35) are given by:

$\mathrm{q}_{\mathrm{d}}(\mathrm{r}, \mathrm{t}=0)=\mathrm{q}\left(\mathrm{p}_{0}, \mathrm{~T}_{0}\right) ; \mathrm{T}_{\mathrm{Sd}}(\mathrm{t}=0)=\mathrm{T}_{0}$

$\frac{\partial \mathrm{q}_{\mathrm{d}}(\mathrm{r}=0, \mathrm{t})}{\partial \mathrm{r}}=0$

$\mathrm{q}_{\mathrm{d}}(\mathrm{r}=\mathrm{d} / 2, \mathrm{t})=\mathrm{q}^{*}\left(\mathrm{p}, \mathrm{T}_{\mathrm{Sd}}\right)$

\section{Determination of heat transfer coefficients}

The fluid-particle heat transfer coefficients, $h_{p D}$ (large particle) and $h_{p d}$ (small particle), as well as the overall heat transfer coefficient in the column wall, $U_{g}$, were determined according to the procedure described by Santos et al. (2015).

\section{NUMERICAL METHOD}

The equations for the column model and adsorbent particles were discretized by the finite volume method (Patankar, 1980; Maliska, 2004). The pressurevelocity coupling was treated using the pressure implicit momentum explicit (PRIME) algorithm (Maliska, 1981), and a staggered arrangement was used to store the variables in the computational grid. The properties at the interfaces of each control volume were evaluated through the weight upwind differencing scheme proposed by Raithby and Torrance (1974). The linear systems were solved by the tridiagonal matrix algorithm. The velocities at the inlet and outlet of the column were calculated through the procedure suggested by Marcondes and Maliska (1999). The volumetric mean concentration and temperature in the solid phase, obtained with the solution of the pellet model for the two types of particles (large and small particles), represent the source terms of mass and energy balances for the gas flow. The nonlinearities and couplings between the equations were solved through an iterative procedure. Based on grid refining tests, 100 volumes were used for the discretization of the column, and 10 volumes were used for the discretization of the two types of pellets. All simulations were performed using a constant time-step of $1 \times 10^{-3} \mathrm{~s}$. The numerical procedure used in the solution of the mathematical model consisted of the following steps:

1) Supply the initial values of the variables $p, u, T_{f}$, $q_{D}, q_{d}, T_{S D}$, and $T_{S d}$.

2) Solve Eqs. (29) and (34) for each control volume of the column and obtain the volumetric mean concentration for the large $\left(q_{D}\right)$ and small particles $\left(q_{d}\right)$.

3) Solve Eqs. (30) and (35) for each control volume of the column and obtain the temperatures for the large $\left(T_{S D}\right)$ and small particles $\left(T_{S d}\right)$.

4) Calculate the pressure field $(p)$ through the continuity equation (PRIME method).

5) Calculate the velocity field $(u)$ through the momentum equation.

6) Calculate the temperature field $\left(T_{f}\right)$ through the energy equation.

7) Calculate the density field $\left(\rho_{f}\right)$ through the ideal gas law.

8) Return to Step 2 and iterate until convergence is reached at the current time level.

9) Progress to the next time level.

The following criterion was used to verify the convergence of the solution in each time-step of the unsteady problem solution: 
$\left|\frac{\varphi_{\mathrm{P}}^{\mathrm{k}+1}-\varphi_{\mathrm{P}}^{\mathrm{k}}}{\varphi_{\max }-\varphi_{\min }}\right| \leq 10^{-5}$

where $\left|\phi_{\max }-\phi_{\min }\right|$ denotes the maximum variation of the density field at the k-th iteration. If Eq. (39) is not satisfied for each control volume, a new iteration is required.

\section{RESULTS AND DISCUSSION}

Before proceeding to the analysis of the numerical results obtained in this study, we present the validation of the computational code developed.

\section{Comparison with experimental results}

To the best of our knowledge, there are no experimental results available in the literature for the problem of methane adsorption dynamics in an activated carbon bed with bidisperse packing. The few works (Greenbank, 1990; Greenbank, 1992; Chang, 1994) do not provide detailed data that can be used for validation of the proposed numerical model. However, we can assess the numerical model developed in this work by comparing the results of the bidisperse model where the small and large particles have the same size, and hence, the bidisperse model should coincide with the monodisperse model.

Malek and Farooq (1997) studied the adsorption and desorption dynamics of methane in an activated carbon bed with monodisperse packing. In their work, experimental breakthrough curves of methane, ethane, and propane on activated carbon and silica gel were determined for three temperature values (299.15, 318.15 , and $338.15 \mathrm{~K})$ and two particle sizes $(0.129$ and $0.258 \mathrm{~cm})$. The gas velocity in the column varied between 1.3 and $4.3 \mathrm{~cm} / \mathrm{s}$, while the feed pressure varied between 1.99 and 6.41 bar. A one-dimensional flow was also employed by the authors to study the adsorption and desorption process in the adsorbent column. To validate our computational code, the numerical results were compared with case study 3 of Malek and Farooq (1997), which investigated the methane adsorption in activated carbon. In this case study, a dilute mixture of methane $(5 \%)$ in helium was considered. The gas pressure and temperature applied at the inlet of the column were 2.4848 bar and 299.15 K, respectively, while the gas velocity was kept constant at $1.56 \mathrm{~cm} / \mathrm{s}$. The diameter of the adsorbent particle used in the column packing was $0.258 \mathrm{~cm}$. A stainless-steel column with internal diameter of $3.5 \mathrm{~cm}$ and $40 \mathrm{~cm}$ in length was considered for this test.

To simulate the experiment performed by Malek and Farooq (1997), an additional mass conservation equation similarto Eq. (20) was used for the methane and helium mixture. For reproduction of the experimental results of the monodisperse bed, $x_{D}=0$ and $\delta=1$ were assumed in the simulations with the bidisperse bed model. In this case, the bidisperse model reproduced the case of a monodisperse bed formed solely by the small particles. Figure 2 shows a comparison between the experimental results for the methane adsorption in activated carbon with monodisperse packing and the numerical results obtained with the bidisperse model. It was observed that the bidisperse model reproduced with reasonable precision the experimental data for methane adsorption in activated carbon with monodisperse packing. The best fit between numerical and experimental data was obtained when the effective mass diffusion coefficient in the particle was equal to $1 \times 10^{-4} \mathrm{~cm}^{2} / \mathrm{s}$. Figure 3 shows the comparison of the

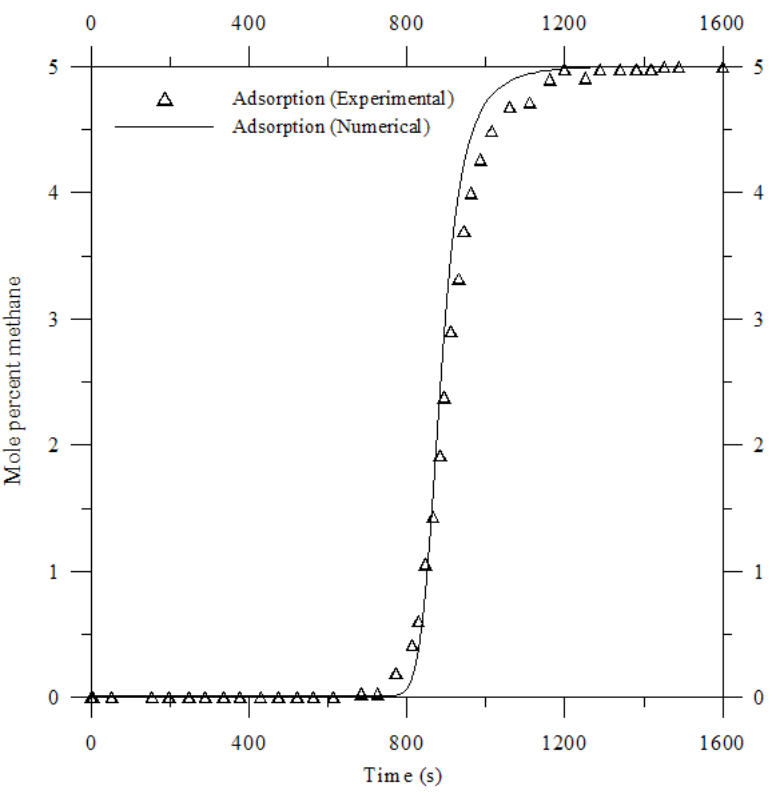

Figure 2. Comparison between experimental and numerical breakthrough curves.

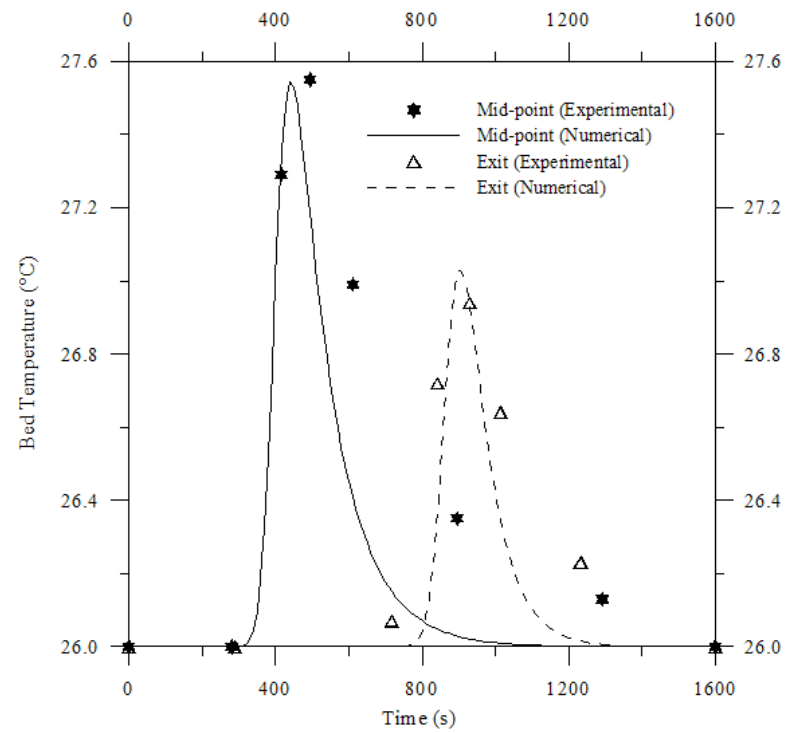

Figure 3. Comparison between experimental and numerical temperature profiles. 
experimental and numerical results in terms of the temperature profile at the middle and at the end of the column during the adsorption test. A reasonable agreement between the results was observed when the overall heat transfer coefficient in the column wall, $\mathrm{U}_{\mathrm{g}}$, was equal to $50 \mathrm{~W} /\left(\mathrm{m}^{2} / \mathrm{K}\right)$. The small differences between the numerical and experimental results observed in Figs. 2 and 3 may be related to the possible radial effects present in the experiment conducted by Malek and Farooq (1997). Similar differences between numerical and experimental results were also obtained by Malek and Farooq (1997).

\section{Numerical results}

In this section, we first evaluate the influence of bidisperse packing on porosity, permeability, and packing density of the bed. Next, the influence of the size ratio of the adsorbent particles on the methane storage capacity and the loading time is investigated. We also analyzed the influence of the inlet gas temperature and the pressure drop in the bed.

The characteristics of the adsorbent column investigated and other data used in the simulation study are shown in Table 1. The compacted adsorbent in the column was G216 carbon manufactured by North American Carbon Coal, Inc. of Columbus, Ohio. The adsorption equilibrium of the $\mathrm{G} 216$ carbon is described by a Langmuir isotherm. The isotherm adjustment parameters were obtained from the experimental data published by Remick and Tiller (1996).

$q^{*}\left(p, T_{s}\right)=\frac{q_{m} b p}{1+b p}$

Table 1. Data and physical properties used in the simulations.

\begin{tabular}{lc}
\hline \multicolumn{1}{c}{ Smaller pellet diameter, $\mathbf{d}$} & $\mathbf{0 , 5}(\mathbf{m m})$ \\
\hline Particle size ratio, $\delta=\mathrm{d} / \mathrm{D}$ & $1 / 20,1 / 15,1 / 10,1 / 5,1$ \\
Intraparticle porosity of the large & 0.6 \\
pellet, $\varepsilon_{\mathrm{pD}}$ & \\
Intraparticle porosity of the small & 0.6 \\
pellet, pd & $0.5(\mathrm{~m})$ \\
Column length, $\mathrm{L}$ & $2.5(\mathrm{~cm})$ \\
Column inner radius, $\mathrm{R}_{\mathrm{i}}$ & $3.5(\mathrm{MPa})$ \\
Initial pressure, $\mathrm{p}$ & $400\left({ }^{\circ} \mathrm{C}\right)$ \\
Initial temperature, $\mathrm{T}_{0}$ & $1.0,7.5,10(\mathrm{kPa})$ \\
Pressure drop, dp= pin-pout & $25,65\left({ }^{\circ} \mathrm{C}\right)$ \\
Inlet temperature, $\mathrm{T}_{\mathrm{in}}$ & $10^{-1}\left(\mathrm{~s}^{-1}\right)^{\mathrm{a}}$ \\
Diffusional time constant, $\mathrm{D}_{\mathrm{ef}} /\left(\mathrm{R}_{\mathrm{p}}\right)^{2}$ & $518.35(\mathrm{~J} / \mathrm{kg} \mathrm{K})$ \\
Ideal gas constant, $\mathrm{Rg}_{\mathrm{g}}$ & $2150\left(\mathrm{~kg} / \mathrm{m}^{3}\right)^{\mathrm{a}}$ \\
Adsorbent density, $\rho_{\mathrm{s}}$ & $648(\mathrm{~J} / \mathrm{kg} \mathrm{K})^{\mathrm{a}}$ \\
Adsorbent specific heat, $\mathrm{C}_{\mathrm{ps}}$ & $0.2(\mathrm{~W} / \mathrm{m} \mathrm{K})^{\mathrm{b}}$ \\
Effective thermal conductivity of the & $2450(\mathrm{~J} / \mathrm{kg} \mathrm{K})^{\mathrm{a}}$ \\
bed, $\lambda_{\mathrm{ef}}$ & $25\left({ }^{\circ} \mathrm{C}\right)$ \\
Sorbate specific heat, $\mathrm{C}_{\mathrm{pg}}$ & $-1.1 \times 10^{6}(\mathrm{~J} / \mathrm{kg})^{\mathrm{a}}$ \\
Surrounding temperature, $\mathrm{T}_{\infty}$ & \\
Adsorption heat, $\Delta \mathrm{H}$ &
\end{tabular}

where

$$
\begin{aligned}
& \mathrm{q}_{\mathrm{m}}=55920 \mathrm{~T}_{\mathrm{s}}^{-2.3} \\
& \mathrm{~b}=1.0863 \times 10^{-7} \exp \left(806 / \mathrm{T}_{\mathrm{s}}\right)
\end{aligned}
$$

Influence of particle size ratio ( $\delta$ ) on porosity, permeability, and packing density

In bidisperse packing, the small particles occupy the voids between the large particles. Consequently, there is a reduction of porosity and an increase in packing density. This fact can be explained with the help of Fig. 4. The porosity and packing density for the bidisperse bed were obtained by applying Eqs. (8) and (19), respectively. The variation in $\delta$ was obtained by fixing the small particle diameter, $d$, and increasing the diameter of the large particle, $D$. As verified by Dias et al. (2008), if $x_{D}=0.7$ (70\% of large particles and $30 \%$ of small particles) for each value of $\delta$, the porosity curve reaches its minimum value. In Fig. 4 , when $\delta$ decreased from 1 (monodispersed bed) to 0.05 , the bed porosity decreased from 0.39 to 0.21 . As a consequence of the reduction in porosity, the packing density increased from 521.79 (monodisperse bed) to $676.17 \mathrm{~kg} / \mathrm{m}^{3}$, which is an increase of $29.57 \%$, relative to the monodisperse bed condition. An increase in packing density is desirable for ANG vessels; however, reduction in porosity may reduce the bed permeability and hence increase loading times. Fig. 5 shows the effect of $\delta$ on the bed permeability. The permeability in the bidisperse bed was obtained from Eq. (24). From Fig. 5, when the size ratio decreased from 1 to 0.05 , the permeability increased from its minimum value $\left(2.75 \times 10^{-10} \mathrm{~m}^{2}\right)$ corresponding to a monodisperse bed condition, reached a maximum of $5.05 \times 10^{-10} \mathrm{~m}^{2}$ for $\delta=0.23$, and decreased to $3.43 \times 10^{-10} \mathrm{~m}^{2}$ when $\delta=$

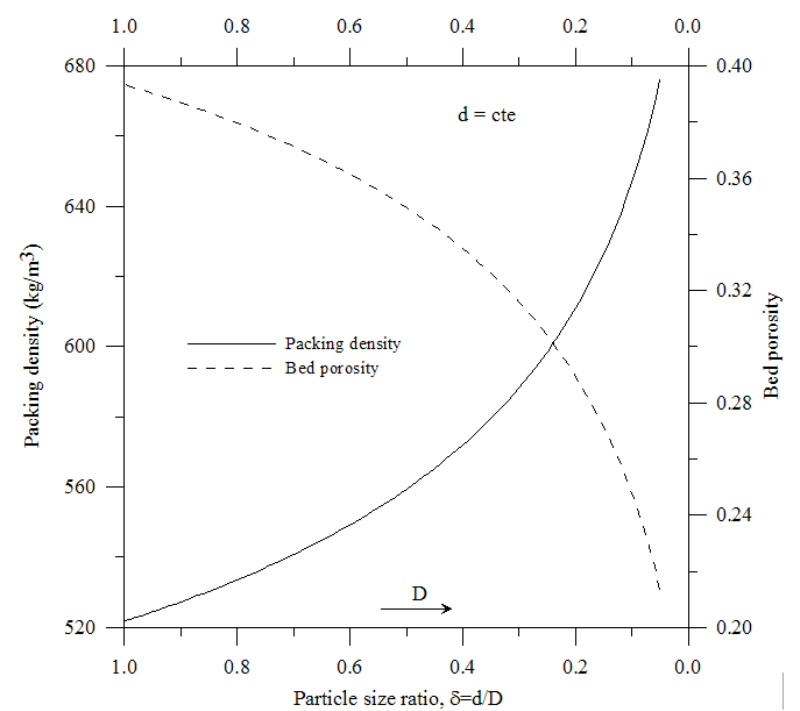

Figure 4. Effect of particle size ratio $\delta$ on porosity and packing density of bed. 


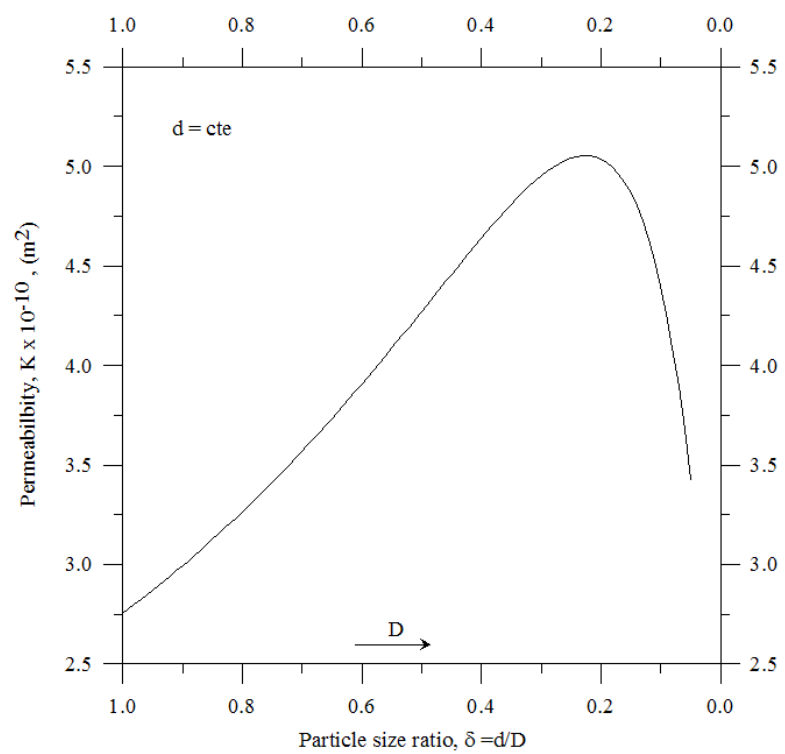

Figure 5. Effect of particle size ratio $\delta$ on bed permeability.

0.05 . Therefore, in the case investigated, the minimum porosity condition did not correspond to the minimum permeability condition as one might expect. This fact was observed by Dias et al. (2007), where the permeability of the bidispersed bed (small porosity) was higher than that of the monodisperse bed (large porosity) for $\delta$ between 1 and 0.1 . According to Dias et al. (2007), we can verify this effect if a monodisperse packing composed of small particles is used to build a bidisperse bed by mixing the small particles with large particles. In such a case, it is possible to increase the packing density with simultaneous increases in permeability and storage capacity without substantially increasing the loading time. However, the choice of the most appropriate value for $\delta$ depends on a rigorous simulation study such as the analysis proposed here.

\section{Influence of particle size ratio $\delta$ on storage capacity and loading time}

We now investigate the influence of particle size ratio on load performance. The following values of $\delta$ were analyzed: 1 (monodisperse bed), 1/5, 1/10, 1/15, and $1 / 20$. The gas pressure and temperature at the inlet of the column were equal to $3.51 \mathrm{MPa}$ and $298.15 \mathrm{~K}$, respectively. The pressure drop imposed on the column was $10 \mathrm{kPa}$. In Fig. 6, the methane storage capacity increased from $111.16 \mathrm{~V} / \mathrm{V}(\delta=1)$ up to $133.83 \mathrm{~V} / \mathrm{V}$ $(\delta=1 / 20)$. This gain of $22.67 \mathrm{~V} / \mathrm{V}$ represents an increase of $20.34 \%$ compared to the monodisperse bed condition. Although this percentage is quite significant, the use of $\delta=1 / 20$ was not feasible because of the high loading time greater than $800 \mathrm{~s}$. Therefore, a better choice that represents a good compromise between storage capacity and load time was $\delta=1 / 10$, where the storage capacity was $129.54 \mathrm{~V} / \mathrm{V}$ and the loading time

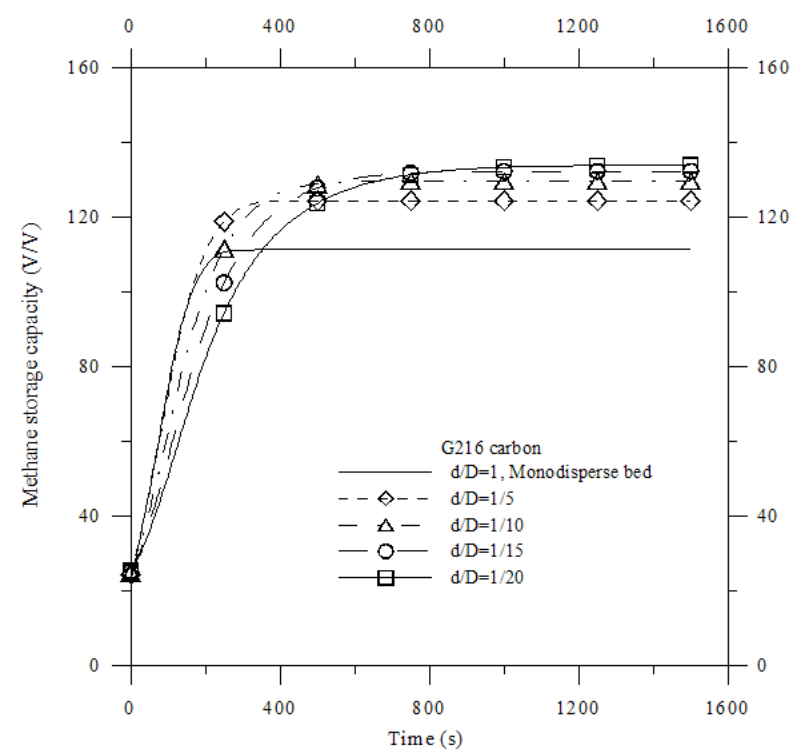

Figure 6. Effect of particle size ratio on methane storage capacity and loading time.

was approximately $500 \mathrm{~s}$. For the last value of $\delta$, the gain in storage capacity relative to the monodisperse bed condition was $18.38 \mathrm{~V} / \mathrm{V}$ or $16.53 \%$. Although the monodisperse bed had the smallest permeability, its loading time was lesser than that of the bidisperse bed configuration. Such behavior is explained because the mass transfer process in the column depends not only on the gas flow resistance between the particles, which is a function of bed permeability, but also on the mass diffusion resistance within the adsorbent particles. As a consequence, a bidisperse bed with large permeability requires more filling time because of the large amount of adsorbent present in the system.

\section{Influence of inlet gas temperature on methane storage capacity}

The adsorption promoted at lower temperatures increased the adsorbed mass and consequently the methane storage capacity. Therefore, in this section, we evaluate the loading performance when the inlet gas temperature is reduced. Two inlet gas temperatures were considered: 338.15 and $298.15 \mathrm{~K}$. The feed pressure was equal to $3.51 \mathrm{MPa}$, while the pressure drop imposed on the column was equal to $10 \mathrm{kPa}$. A comparison between the load performance obtained with the monodisperse and bidisperse beds was performed. For the bidisperse bed, the particle size ratio $\delta$ was equal to $1 / 10$. In Fig. 7, the solid lines represent the loading process of the bidisperse bed, while the dotted lines represent the process of the monodisperse bed. With respect to Fig. 7, when the inlet temperature was reduced from $338.15 \mathrm{~K}$ to 298.15 $\mathrm{K}$ for the bidisperse case, the methane storage capacity in the column increased $31.52 \mathrm{~V} / \mathrm{V}$. However, for the monodisperse bed, the increase in storage capacity 


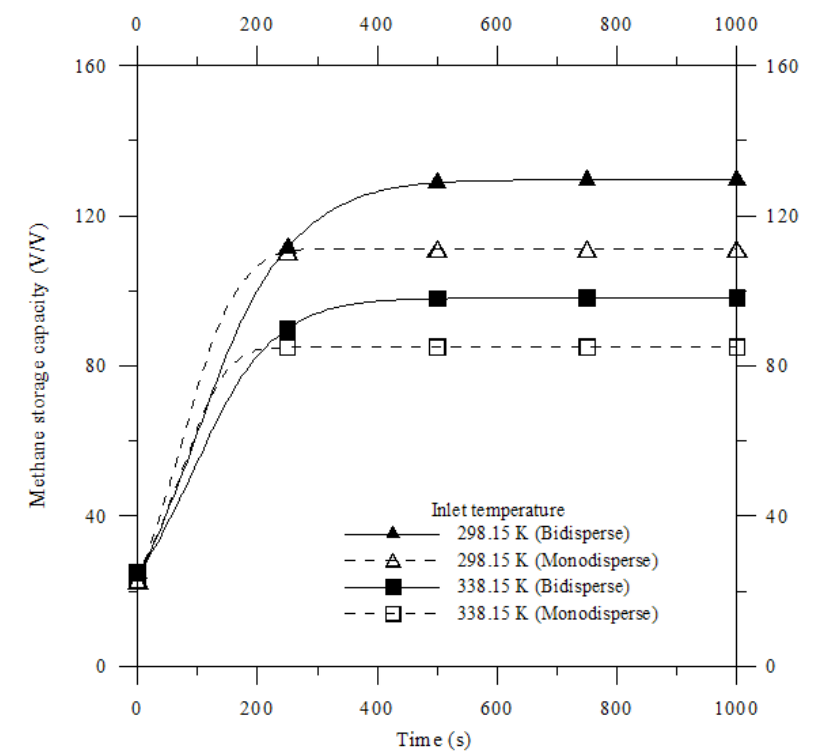

Figure 7. Effect of inlet gas temperature on methane storage capacity.

was only $26.23 \mathrm{~V} / \mathrm{V}$. In addition, for the same inlet gas temperature, the bidisperse model had a larger storage capacity compared to the monodisperse model.

\section{Influence of pressure drop on loading time}

Previous studies performed by Santos et al. (2009) and Santos et al. (2015) demonstrated that increasing the pressure drop imposed on the adsorbent column reduces the loading time. Therefore, this case study investigated the impact of the applied pressure drop on the loading time when monodisperse and bidisperse beds were used in ANG vessels. For the bidisperse bed, the particle size ratio employed was $1 / 10$. Three different values of pressure drop were used in the analysis: $1,7.5$, and $10 \mathrm{kPa}$. The inlet gas temperature was $298.15 \mathrm{~K}$, while the pressure at the outlet of the column was assumed to be $3.5 \mathrm{MPa}$. The feed pressure is the sum of the outlet pressure with the pressure drop applied to the column. Figure 8 shows the charging process for both monodisperse and bidisperse beds for different values of pressure drop imposed on the column. The solid lines represent the bidisperse bed, while the dotted lines represent the monodisperse bed. Regardless of the type of packing, when the pressure drop was small $(1.0 \mathrm{kPa})$, the gas velocity was also small and the charge time was large. By progressively increasing the applied pressure drop, the flow velocity increased and the charge time decreased for both the monodisperse and bidisperse beds. However, it was observed that a convergence occurred in the loading curves in both cases when the applied pressure drop approached $10 \mathrm{kPa}$. This suggests that there was no substantial reduction in charge time when the applied pressure drop was greater than $10 \mathrm{kPa}$. This can be explained by the fact that the mass transfer in the

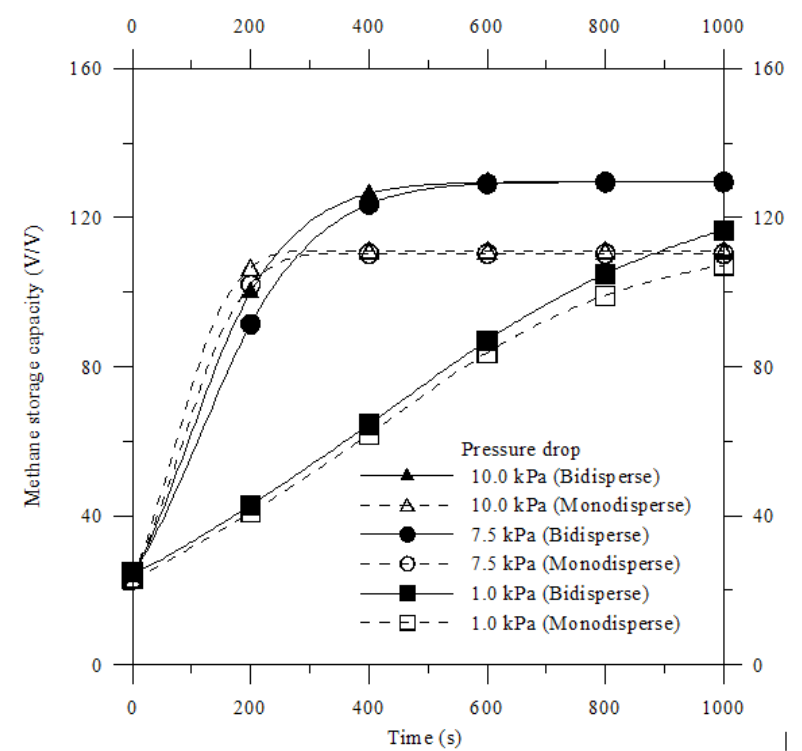

Figure 8. Effect of pressure drop on loading time.

column depends on the gas velocity outside the solid phase and the mass diffusion in the adsorbent particles. By progressively increasing the pressure drop, the gas velocity becomes much larger than the rate of diffusion of the gas in the adsorbent particles. When this happens, the resistance to the mass transfer out of the adsorbent decreases, but the mass transfer into the column is dominated by the mass diffusion process of the adsorbent; for this situation, there is no possibility of reducing the loading time by elevating the applied pressure drop. Compared to the monodisperse bed case, the large charge time required by the bidisperse bed resulted from the large amount of adsorbent present in the system.

\section{CONCLUSIONS}

The present work provided a numerical study of methane adsorption dynamics in activated carbon beds with bidisperse packing. The bidisperse packing was tested to reduce the void spaces between the adsorbent particles and to improve the gas storage capacity in the porous bed. The modeling of the loading process was developed using the mass, momentum, and energy balances along with the ideal gas law. The finite volume method was applied to solve the coupled non-linear system of equations. The effect of bidisperse packing on the methane storage and charge time was investigated. Compared with the monodisperse bed condition, the results obtained showed that bidisperse packing can increase packing density by up to $30 \%$ and volumetric storage capacity by up to $20 \%$. However, when the size ratio $\delta$ applied to the bidisperse bed was small $(\delta=1 / 20)$, the required loading time increased considerably (more than $800 \mathrm{~s}$ ). For the case investigated, the use of $\delta=1 / 10$ showed a 
good compromise between storage capacity (16\% gain over the monodisperse bed) and required loading time (about $500 \mathrm{~s}$ ). Regardless of the type of packing used (monodisperse or bidisperse), the load performance can be optimized by reducing the gas inlet temperature, as well as by raising the pressure drop applied to the bed. According to the present investigation, bidisperse packing can contribute significantly to the improved performance of ANG vessels.

\section{NOMENCLATURE}

$C_{p} \quad$ Specific heat at constant pressure $(\mathrm{J} / \mathrm{kg} \mathrm{K})$

$C_{S} \quad$ Volumetric heat capacity of the adsorbent $\left(\mathrm{J} / \mathrm{m}^{3} \mathrm{~K}\right)$

$d \quad$ Diameter of the small pellet (m)

$d_{a v} \quad$ Average diameter of the pellets (m)

$D \quad$ Diameter of the large pellet (m)

$D_{e f} \quad$ Effective mass diffusion coefficient $\left(\mathrm{m}^{2} / \mathrm{s}\right)$

$q$ Concentration of the gas in the adsorbent $\left(\mathrm{kg} / \mathrm{m}^{3}\right)$

$q \quad$ Volumetric average concentration over the pellet $\left(\mathrm{kg} / \mathrm{m}^{3}\right)$

$u \quad$ Intersticial velocity $(\mathrm{m} / \mathrm{s})$

$h_{p} \quad$ Fluid-particle heat transfer coefficient $\left(\mathrm{W} / \mathrm{m}^{2} \mathrm{~K}\right)$

$\Delta H \quad$ Heat of adsorption $(\mathrm{J} / \mathrm{kg})$

$L \quad$ Column length (m)

$\mathrm{Nu} \quad$ Nusselt number

$p \quad$ Gas pressure $(\mathrm{Pa})$

$K \quad$ Bed permeability $\left(\mathrm{m}^{2}\right)$

$\mathrm{Pr} \quad$ Prandtl number

$r \quad$ Pellet radial coordinate $(\mathrm{m})$

$R \quad$ Column radius (m)

$R e \quad$ Reynolds number

$R_{g} \quad$ Ideal gas constant $(\mathrm{J} / \mathrm{kg} \mathrm{K})$

$t^{g} \quad$ Time (s)

$T \quad$ Temperature (K)

$U_{g} \quad$ Overall heat transfer coefficient $\left(\mathrm{W} / \mathrm{m}^{2} \mathrm{~K}\right)$

$x \quad$ Axial coordinate in the column (m)

$x_{D} \quad$ Volumetric fraction of the large pellet

\section{Greek letters}

$\varepsilon \quad$ Bed porosity (interparticle)

$\varepsilon_{D} \quad$ Fractional porosity of the large pellet

$\varepsilon_{d} \quad$ Fractional porosity of the small pellet

$\varepsilon_{p D} \quad$ Porosity of the large pellet (intraparticle)

$\varepsilon_{p d} \quad$ Porosity of the small pellet (intraparticle)

$\lambda^{p d} \quad$ Thermal conductivity $(\mathrm{W} / \mathrm{m} \mathrm{K})$

$\rho \quad$ Density $\left(\mathrm{kg} / \mathrm{m}^{3}\right)$

$\tau \quad$ Bed tortuosity

$\mu \quad$ Gas viscosity $(\mathrm{kg} / \mathrm{m} \mathrm{s})$

$\delta \quad$ Particle size ratio $(\delta=\mathrm{d} / \mathrm{D})$

\section{Subscripts}

$\infty \quad$ Relative to the ambient

0 Relative to the initial condition

$\begin{array}{ll}d & \text { Small pellet } \\ D & \text { Large pellet } \\ e & \text { Relative to the external surface } \\ f & \text { Relative to the fluid phase } \\ \text { in } & \text { Relative to the column inlet } \\ p & \text { Relative to the pellet } \\ S & \text { Relative to the solid phase } \\ w & \text { Relative to the column wall }\end{array}$

\section{Superscripts}

Relative to the adsorption equilibrium

\section{ACKNOWLEDGMENTS}

The authors thank the CNPq (The National Council for Scientific and Technological Development of Brazil) and FUNCAP (The Foundation to Support the Scientific and Technological Development of CearáBrazil) for their financial support through grants (308865/2016-2 and 305851/2015-2) and (BP1-006700162.01.00/12), respectively.

\section{REFERENCES}

Bem Aim, R., LeGoff, P., Le Lec, P. La perméabilité de milieux poreux formés par empilement de mélanges binaires de grains sphériques. Powder Technol., 5, 51-60 (1971). https://doi.org/10.1016/00325910(71)80070-0

Chang, C. H. Packing adsorbent particles for storage of natural gas. U.S. Patent 5,308,821, (1994).

Chang, K. J., Talu, O. Behavior and performance of adsorptive natural gas storage cylinders during discharge. Appl. Therm. Eng. 16, 359-374 (1996). https://doi.org/10.1016/0032-5910(71)80070-0

Dias, R. P., Fernandes, C. S., Teixeira, J. A., Mota, M. G., Yelshin, A. I. Particulate Binary Mixtures: Dependence of Packing Porosity on Particle Size Ratio. Ind. Eng. Chem. Res. 43, $7912-7919$ (2004). https://doi.org/10.1021/ie040048b

Dias, R. P., Fernandes, C. S., Teixeira, J. A., Mota, M. G., Yelshin, A. I. Permeability analysis in bisized porous media: Wall effect between particles of different size. Journal of Hydrology 349, 470-474 (2008). https://doi.org/10.1016/j. jhydrol.2007.11.020

Dias, R. P., Fernandes, C. S., Mota, M., Teixeira, J. A., Yelshin, A. Permeability and effective thermal conductivity of bisized porous media. International Journal of Heat and Mass Transfer, 50, 1295-1301 (2007). https://doi.org/10.1016/j. ijheatmasstransfer.2006.09.039

Greenbank, M. Preparation of a dense pack particulate gas adsorbent. U.S. Patent 4,972,658, (1990).

Greenbank, M. Method and means for improved gas adsorption. U.S. Patent 5,094,736, (1992). 
Kayal, S., Sun, B., Chakraborty, A. Study of metalorganic framework MIL-101 (Cr) for natural gas (methane) storage and compare with other MOFs (metal-organic frameworks). Energy, 91, 772-781 (2015).https://doi.org/10.1016/j.energy.2015.08.096

Leitzelement, M., Lo, C. S., Dodds, J. Porosity and permeability of ternary mixtures of particles. Powder Technol., 41, 159-164 (1985). https://doi. org/10.1016/0032-5910(85)87034-0

Lozano-Castelló, D., Monge-Alcañiz, J., Casa-Lillo, M. A., Cazorla-Amorós, D. Advances in the study of methane storage in porous carbonaceus materials. Fuel, 81, 1777-1803 (2002). https://doi. org/10.1016/S0016-2361(02)00124-2

MacDonald, M. J., Chu, C. F., Guilloit, P. P., Ng, K. M.A generalized Blake-Kozeny equation for multisized spherical particles. AIChE J., 37, 1583-1588 (1991). https://doi.org/10.1002/aic.690371016

Malek, A., Farooq, S. Kinetics of Hydrocarbon Adsorption on Activated Carbon and Silica Gel. AIChE J., 43, 761-776 (1997). https://doi. org/10.1002/aic.690430321

Maliska, C. R. A solution method three-dimensional parabolic fluid flow problems in nonorthogonal coordinates. Ph. D. Thesis, University of Warterloo, Warterloo, Canada, (1981).

Maliska, C. R. Computational Fluid Mechanics and Heat Transfer. LTC, Rio de Janeiro, Brazil, (2004) (in Portuguese).

Marcondes, F., Maliska, C. R. Treatment of the inlet boundary conditions in natural convection flows in open-ended channels. Num. Heat Transf. Part B, 35, 317-345 (1999). https://doi. org/10.1080/104077999275893

Menon, V. C., Komarneni, S. Porous adsorbents for vehicular natural gas storage: A review. J. of Porous Mater., 5, 43-58 (1998). https://doi. org/10.1023/A:1009673830619

Mota, J. P. B., Esteves, I. A. C. C., Rostam-Abadi, M. Dynamic modeling of an adsorption storage tank using a hybrid approach combining computational fluid dynamics and process simulation. Computers and Chem. Eng., 28, 2421-2431 (2004). https://doi. org/10.1016/j.compchemeng.2004.06.004

Mota, J. P. B., Rodrigues, A. E., Saatdjian, E., Tondeur, D. Dynamics of natural gas adsorption storage systems employing activated carbon. Carbon, 35, 1259-1270 (1997). https://doi.org/10.1016/S00086223(97)00075-4

Mota, J. P., Lyubchik, S. Recent Advances in Adsorption Processes for Environmental Protection. Springer (2008). https://doi.org/10.1007/978-1-4020-6805-8

Mota, M., Teixeira, J. A., Yelshin, A. Binary spherical particle mixed beds porosity and permeability relationship measurement. Trans. Filtration Soc., 1, 101-106 (2001)
Patankar, S. V. Numerical Heat Transfer and Fluid Flow. Hemisphere, New York, (1980).

Prajwal, B. P., Ayappa, K. G. Evaluating methane storage targets: from powder samples to onboard storage systems. Adsorption, 20, $769-776$ (2014). https://doi.org/10.1007/s10450-014-9620-1

Rahman, K. A., Loh, W. S., Chakraborty, A., Saha, B. B., Chun, W. G. Thermal enhancement of charge and discharge cycles for adsorbed natural gas storage. Appl. Therm. Eng., 31, 1630-1639 (2011). https:// doi.org/10.1016/j.applthermaleng.2011.02.002

Raithby, G. D., Torrance, K. E. Upstream-Weight Differencing Schemes and their application to elliptic problems involving fluid flows. Computers \& Fluids, 2, 191-206 (1974). https://doi. org/10.1016/0045-7930(74)90013-9

Remick, R. J., Tiller, A. J. Heat generation in natural gas adsorption systems, Gaseous Fuels for Transportation International Conference. Vancouver, Canada, (1996).

Rios, R. B., Bastos-Neto, M., Amora Jr., M. R., Torres, A. E. B., D. C. S., Azevedo, D. C. S., Cavalcante Jr., C. L. Experimental analysis of the efficiency on charge/discharge cycles in natural gas storage by adsorption. Fuel, 90, 113-119 (2011). https://doi. org/10.1016/j.fuel.2010.07.039

Roszak, E. A., Chorowski, M. Exergy analysis of combined simultaneous liquid natural gas vaporization and adsorbed natural gas cooling. Fuel, 111, 755-762 (2013). https://doi.org/10.1016/j.fuel.2013.03.074

Santos, J. C., Gurgel, J. M., Marcondes, F. Numerical simulation of fast charge of natural gas on activated carbon in conjunction with variable velocity. Appl. Therm. Eng., 90, 258-265 (2015). https://doi. org/10.1016/j.applthermaleng.2015.05.091

Santos, J. C., Gurgel, J. M., Marcondes, F. Analysis of a new methodology applied to the desorption of natural gas in activated carbon vessels. Appl. Therm. Eng., 73, 929-937 (2014). https://doi. org/10.1016/j.applthermaleng.2014.08.046

Santos, J. C., Marcondes, F., Gurgel, J. M. Performance analysis of a new tank configuration applied to the natural gas storage systems by adsorption. Appl. Therm. Eng., 29, 2365-2372 (2009). https://doi. org/10.1016/j.applthermaleng.2008.12.001

Standish, N., Collins, D. N. The permeability of ternary particulate mixtures for laminar flow. Powder Technol., 36, 55-60 (1983). https://doi. org/10.1016/0032-5910(83)80008-4

Standish, N., Mellor, D. G. The permeability of ternary coke mixtures. Powder Technol., 27, 61-68 (1980). https://doi.org/10.1016/0032-5910(80)85042-X

Talu, O. An overview of adsorptive storage of natural gas. Proceedings of International Conference on Fundamentals of Adsorption, Kyoto, Japan, 655-662 (1992). https://doi.org/10.1016/S01672991(08)63573-9 
Vasiliev, L. L., Kanonchik, L. E., Mishkinis, D. A., Rabetsky, M. I. Adsorbed natural gas storage and transportation vessels. International J. Therm. Sci., 39, 1047-1055 (2000). https://doi.org/10.1016/ S1290-0729(00)01178-9
Yang, X. D., Zheng, Q. R., Gu, A. Z., Lu, X. S. Experimental studies of the performance of adsorbed natural gas storage system during discharge. Appl. Therm. Eng., 25, 591-601 (2005). https://doi. org/10.1016/j.applthermaleng.2004.07.002 
